REVIEW

\title{
Important cutaneous manifestations of inflammatory bowel disease
}

\author{
L B Trost, J K McDonnell
}

Postgrad Med J 2005;81:580-585. doi: 10.1136/pgmj.2004.031633

Inflammatory bowel disease (IBD) has many extraintestinal manifestations. Cutaneous manifestations are usually related to the activity of the bowel disease but may have an independent course. Anyone presenting with IBD should be examined for cutaneous manifestations. Pyoderma gangrenosum is a severe painful ulcerating disease that requires moist wound management and, in the absence of secondary infection, systemic corticosteroids, cyclosporine, or both. Infliximab may also be used. Erythema nodosum is a common cause of tender red nodules of the shins. Management includes leg elevation, NSAIDs, and potassium iodide. Oral manifestations of IBD include aphthous stomatitis, mucosal nodularity (cobblestoning), and pyostomatitis vegetans. Treatment should be directed both at the cutaneous lesions and at the underlying systemic condition.

See end of article for authors' affiliations

Correspondence to: Dr L B Trost, Department of Dermatology, A61, Cleveland Clinic Foundation, 9500 Euclid Avenue, Cleveland, Ohio 44195, USA; TrostL@ccf. org

Submitted

14 December 2004

Accepted 4 January 2005 nflammatory bowel disease (IBD) is a common clinical entity that affects $0.37 \%$ of the population. ${ }^{1}$ Its two main subtypes, Crohn's disease (CD) and ulcerative colitis (UC), are commonly associated with abdominal pain, diarrhoea, rectal bleeding, weight loss, and signs of malnutrition. ${ }^{2}$

IBD is associated with a wide array of extraintestinal manifestations, including cutaneous, musculoskeletal, hepatobiliary, ocular, and metabolic conditions (see box 1). One third of patients with IBD will develop an extraintestinal manifestation. ${ }^{3}$ Of those patients, up to one third will develop cutaneous manifestations. ${ }^{45}$

Patients presenting with IBD should be examined thoroughly for cutaneous manifestations. This article will discuss the epidemiology, pathophysiology, diagnosis, and management of important cutaneous manifestations of IBD.

\section{PYODERMA GANGRENOSUM \\ Epidemiology and pathophysiology}

Pyoderma gangrenosum (PG) is a severe ulcerating non-infectious neutrophilic dermatosis. UC is the most common underlying disease associated with PG in adults. ${ }^{6} \mathrm{PG}$ has been reported in $1 \%$ to $10 \%$ of patients with UC and in $0.5 \%$ to $20 \%$ of patients with $\mathrm{CD} .^{7}$ It occurs equally in men and women with a peak age incidence between 25 and 54 years. ${ }^{8}$ Some $50 \%-78 \%$ of patients with PG have an underlying systemic disease ${ }^{9}$ such as IBD, myeloproliferative disease, and rheumatological disease. ${ }^{10}$ While the pathophysiology is not completely understood, it is thought to involve impaired cellular immunity and abnormal neutrophil function. ${ }^{11}$

\section{Presentation}

Four variants of PG have been described: ulcerative, pustular, bullous, and vegetative. Ulcerative and pustular PG are associated with IBD, arthritis, and malignancy, whereas bullous PG is associated with myeloproliferative disorders. Vegetative PG usually has no associated underlying systemic conditions. PG can occur before, during, or after the onset of IBD, and both diseases can occur independently of one another. ${ }^{8}$ Classically, PG begins with pain, followed by pustule formation and rapid ulceration. A sterile, purulent necrotic centre remains and is surrounded by a bluish border. It most commonly occurs on the lower extremities although it can occur on any part of the body (see fig 1). ${ }^{12}{ }^{13}$ About half of patients with PG develop large ulcers in response to minor trauma (for example, venipuncture and surgical debridement). ${ }^{9}$

\section{Diagnosis}

PG is a diagnosis of exclusion. Box 2 lists the differential diagnosis of PG. ${ }^{9}$ It is vital to exclude infectious aetiologies because infection may preclude the use of systemic corticosteroids and other immunosuppressants. Underlying systemic diseases must be identified and treated. ${ }^{14}$

A detailed history should address exposure to syphilis, ingestion of iodide or bromide, and recent spider bites. Routine testing for bacteria, mycobacteria, and deep fungal infection from tissue cultures is recommended. Additional studies may include syphilis serology, antiphospholipid antibody levels, serum protein electrophoresis, immunoelectrophoresis, and serum iodide and bromide levels. ${ }^{8}$ Tissue should be examined histologically to rule out other diseases. Currently, specific laboratory or histopathological tests for PG are not available. ${ }^{15}$ In classic ulcerative PG, there is neutrophilic infiltrate centrally in the ulcer and lymphocytic infiltrate in the periphery. ${ }^{8}$

\section{Management}

Management should be directed at both the lesions of $\mathrm{PG}$ and at the underlying disorder. ${ }^{4}$ To date, almost all reports of efficacy have come from case series and case reports rather than randomised prospective controlled trials.

Abbreviations: IBD, inflammatory bowel disease; $C D$, Crohn's disese; UC, ulcerative colitis; PG, pyoderma gangrenosum; EN, erythema nodosum 
Box 1 Common extraintestinal manifestations of inflammatory bowel disease ${ }^{64 *}$

\section{Cutaneous}

- Specific lesions: fissures and fistulas, aphthous stomatitis, mucosal nodularity (cobblestoning), pyostomatitis vegetans, metastatic Crohn's disease

- Reactive lesions: erythema nodosum, pyoderma gangrenosum, aphthous stomatitis, vesiculopustular eruptions, necrotising vasculitis, cutaneous polyarteritis nodosa

- Miscellaneous: epidermolysis bullosa acquisita, vitiligo, psoriasis, secondary amyloidosis, bowel associated dermatosis-arthritis syndrome

- Cutaneous manifestations secondary to nutritional malabsorption: acrodermatitis enteropathica (zinc), scurvy (vitamin C), purpura (vitamin C and K), pellagra (niacin), stomatitis-glossitis-angular cheilitis (vitamin B), non-specific eczema and dry skin (essential fatty acids), abnormal hair and nails (protein)

- Cutaneous manifestations secondary to treatment: drug eruption, peristomal dermatitis

\section{Musculoskeletal}

- Arthritis, osteoporosis, hypertrophic osteoarthropathy

\section{Hepatobiliary}

- Primary sclerosing cholangitis, autoimmune hepatitis, cirrhosis, fatty liver, hepatic granulomas in Crohn's disease

\section{Ocular}

- Uveitis (iritis), episcleritis, scleromalacia

\section{Metabolic}

- Growth retardation in children and adolescents, delayed sexual maturation

*Modified from Das 1999 and Gregory and Ho 1992.
Lesion specific management can include topical, intralesional, and systemic therapies. Moist wound management with an emphasis on preventing secondary infections is crucial. Hydroactive dressings, foam dressings, and laminate dressings are commonlyly used. Hydrogen peroxide is not recommended because of wound cell toxicity. ${ }^{10}$ Topical sodium cromoglyate has been reported to be effective with and without systemic corticosteroid therapy. ${ }^{16}$ Topical corticosteroid therapy has been successful in treating peristomal $\mathrm{PG}^{17}$ while in non-peristomal skin, local injections of triamcinolone acetonide $10-40 \mathrm{mg} / \mathrm{ml}$ with and without systemic corticosteroids have been used. ${ }^{18}{ }^{19}$ If secondary infection occurs, topical and intralesional corticosteroid therapy is contraindicated. ${ }^{10}$

Prednisone is generally considered the drug of choice. Initial doses range from 1 to $2 \mathrm{mg} / \mathrm{kg} /$ day. ${ }^{9} 142021$ Cyclosporine at doses ranging from 3 to $5 \mathrm{mg} / \mathrm{kg} / \mathrm{day}$ has been shown to be effective in case reports both alone and in combination with systemic corticosteroids and should be considered in refractory cases of PG. ${ }^{1422-28}$ Sulfa drugs (for example, sulfasalazine, sulfapyridine, and sulfamethoxypyridazine) are also commonly used. Pulse methylprednisolone treatment has had mixed results in various reports. Other systemic agents that have either limited or conflicting results include dapsone, clofazimine, minocycline, azathioprine, alkylating agents, FK506 (tacrolimus), ${ }^{14}$ and cyclophosphamide. ${ }^{7}$ A recent retrospective review of 86 patients with PG showed that the average length of treatment with systemic agents was 11.5 months, and although most lesions healed within one year, 95\% had remitted in three years. ${ }^{9}$

In the past few years infliximab, a chimeric anti-TNF $\alpha$ monoclonal antibody, has shown great promise in treating refractory PG associated with CD. Infliximab is currently approved by the United States Food and Drug Administration for the treatment of rheumatoid arthritis and CD, but its efficacy has been reported in a wide range of inflammatory conditions. ${ }^{29}$ In the treatment of refractory PG associated with $\mathrm{CD}$, favourable responses have been reported to occur within 12 hours to one week, with complete healing occurring in 1 to 11 weeks. In most reports, infliximab was given at a dose of $5 \mathrm{mg} / \mathrm{kg}$, and treatments were spaced out

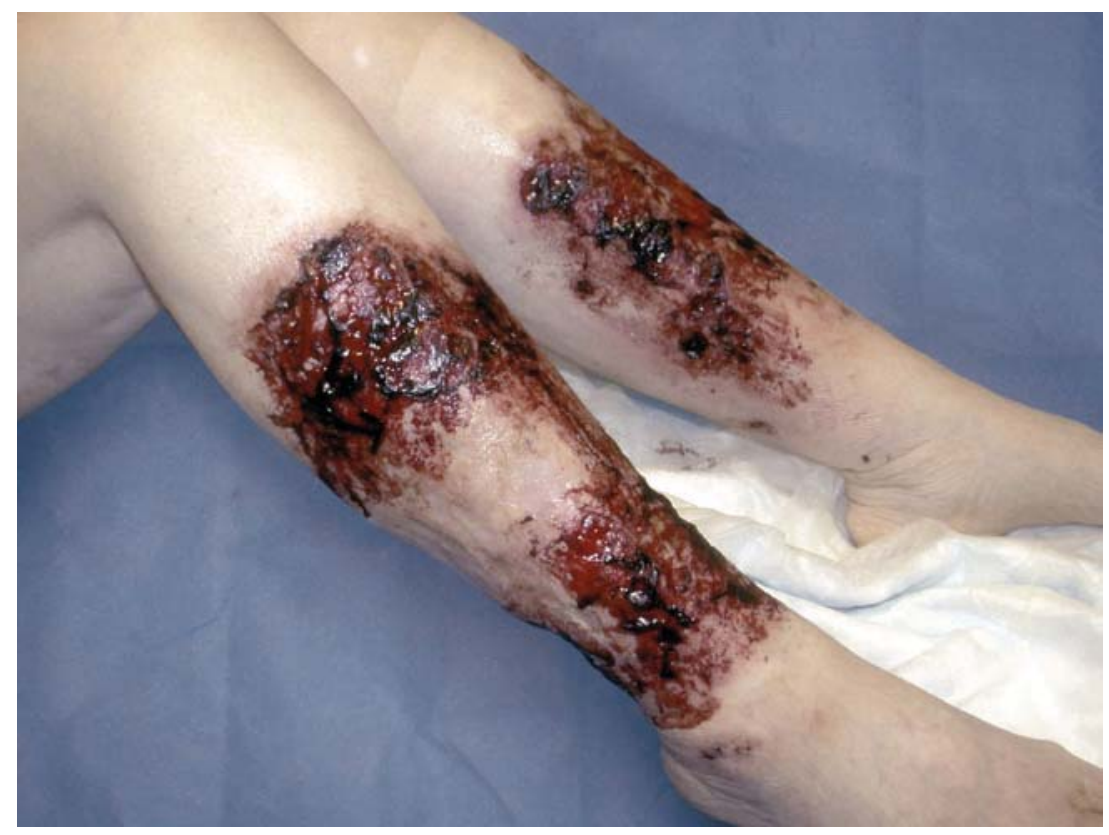

Figure 1 Pyoderma gangrenosum. Painful and irregularly shaped ulcers with undermined borders. 
Box 2 Differential diagnosis of pyoderma gangrenosum ${ }^{9 *}$

\section{Infection}

- Bacterial infection

- Mycobacterial infection

- Fungal infection

- Parasitic infection

- Viral infection

Sweet's syndrome Spider bite

- Brown recluse spider

Malignancy

- Squamous cell carcinoma

- Basal cell carcinoma

- Cutaneous T cell lymphoma

Halogenoderma

Factitial ulceration

Vascular disease

- Venous or arterial insufficiency

- Antiphospholipid antibody associated occlusive disease

- Thrombophlebitis with gangrene

Systemic disease

- Systemic lupus erythematosus

- Rheumatoid arthritis

- Behçet's disease

- Wegener's granulomatosis

*Modified from Bennett, et al 2000.

several weeks apart. ${ }^{30-34}$ However, some cases showed only temporary improvement or no improvement at all. ${ }^{31}$

Although surgery can cause exacerbations of PG, cutaneous surgery has been shown to be effective at inducing disease remission, but it must be performed in conjunction with immunosuppressive therapies in patients with remitting or stable disease. Options include split-skin grafts and autologous keratinocyte grafts. ${ }^{10}$ Because the course of PG can be independent of the course of IBD and has even been reported years after proctocolectomy, bowel resection is not a primary therapy. ${ }^{35} 36$

\section{ERYTHEMA NODOSUM}

\section{Epidemiology and pathophysiology}

Erythema nodosum (EN) is the most common cause of lower extremity inflammatory nodules and has been associated with IBD in $11 \%$ of cases, occurring more frequently in patients with UC than $\mathrm{CD}^{37}$ It occurs in women three to six times more frequently than in men and has a peak age incidence between 20 and 30 years. $^{38}$ EN is most commonly associated with streptococcal infections, ${ }^{39}$ medications, sarcoidosis, autoimmune disorders, and IBD (see box 3 )..$^{40}$ The precise aetiology is thought to be a hypersensitivity response involving immune complex deposition in and adjacent to venules in the septa of connective tissue in subcutaneous fat. ${ }^{38}$

\section{Presentation}

EN is characterised by the sudden onset of multiple, bilateral, symmetric, red, warm, and painful nodules about $2 \mathrm{~cm}$ in
Box 3 Common conditions associated with

erythema nodosum ${ }^{40 *}$

\section{Infections}

- Streptococcus $\beta$ haemolyticus infection

- Primary tuberculosis

- Yersinia, salmonella, coccidioidomycosis, and heliobacter infections

\section{Systemic diseases}

- Sarcoidosis

- Inflammatory bowel disease (Crohn's disease and ulcerative colitis)

- Behçet's disease

- Connective tissue diseases

- Sweet's syndrome

\section{Malignancies}

- Haematological neoplasms (lymphoma)

Drugs

- Oral contraceptives

- Penicillins

- Sulfonamides

- Bromides

- lodides

- Analgesics

Pregnancy

*Modified from Gonzales-Gay, et al 2001.

diameter. These lesions most commonly occur on the shins but may also occur on the calves, trunk, and face (see fig 2). Systemic symptoms such as fever, malaise, and joint pain often occur. The typical course lasts for three to six weeks, but the residual bruise-like lesions can last for months. Neither ulceration nor scarring occurs in EN. ${ }^{37}$ In patients with IBD, eruptions of EN often are associated with exacerbations of the bowel disease $\mathrm{e}^{7}$ but not with the severity or extent. ${ }^{41}$

\section{Diagnosis}

A search for an associated disease should always accompany the diagnosis of EN. Although an extensive examination is not needed, both common and serious underlying conditions should be considered. A complete history should include the duration of symptoms and previous similar episodes, history of drug intake (especially oral contraceptives), pregnancy, diarrhoea, and recent pharyngitis, tonsillitis, or upper respiratory tract infection (URI) in the past three weeks. Physical examination findings of arthritis or periarticular ankle inflammation may be indicative of acute sarcoidosis. Also, lymphadenopathy is often found in malignancies such as non-Hodgkin's lymphoma. ${ }^{40}$ Tuberculosis and coccidioidomycosis should be considered in high risk groups. ${ }^{42}$

Laboratory tests should be used depending on the degree of clinical suspicion. Reasonable initial tests include complete blood count (white blood cells with a high percentage of neutrophils and bands often occur), ACE level, ASO titre, and chest radiography.$^{40} \mathrm{~A}$ tuberculin skin test and blood samples for bacterial, virological, fungal, and protozoal infections that are endemic to the area may also be appropriate..$^{38}$ If a biopsy is taken, it should be excisional so that the subcutaneous adipose tissue is included in the specimen. ${ }^{42}$ 


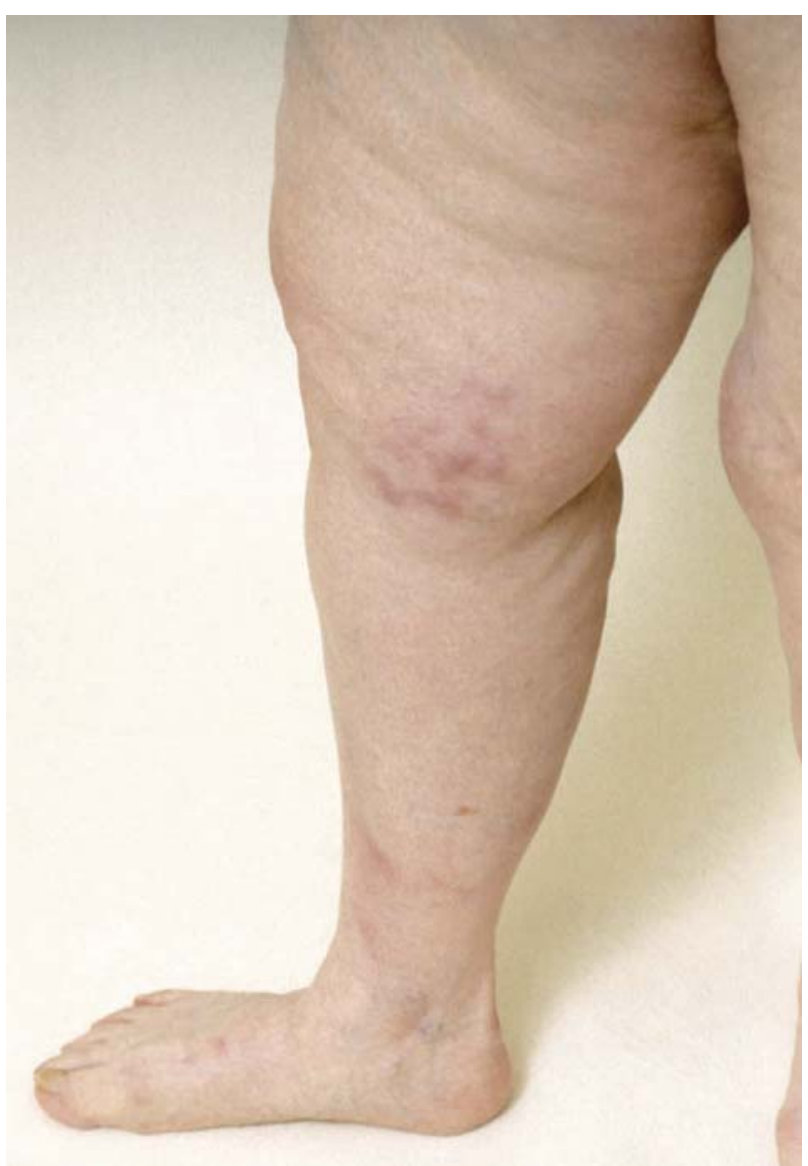

Figure 2 Erythema nodosum. Multiple red, warm, and tender nodules on the leg.

\section{Management}

In most cases, EN is self limiting and will resolve in three to six weeks without scar formation. However, late stage bruising, arthralgias, and joint stiffness may last for months. Recurrences are rare. ${ }^{37} 3843$ When associated with IBD, EN usually will resolve with control of the IBD and often will recur with exacerbations of the bowel disease. ${ }^{37}$ In a study of 792 patients with IBD, every case of EN (48 patients) responded to medical treatment of the IBD. ${ }^{21}$

Management should be both supportive and directed at the underlying associated condition. Supportive treatment includes leg elevation, support stockings, and bed rest. ${ }^{44}$ Aspirin and NSAIDs may also help with pain control and healing. Naproxen ${ }^{45}$ and indomethacin ${ }^{46} 47$ have been reported to be effective in cases refractory to aspirin and NSAID therapy. In addition, potassium iodide has been reported to be successful. ${ }^{37}$ 48-50

Systemic corticosteroids are effective as well. However, it is crucial to rule out underlying infections such as tuberculosis and coccidioidomycosis to prevent dissemination. ${ }^{42}$ Other less common systemic treatments include colchicine and hydroxychloroquine. ${ }^{38}$

\section{ORAL MANIFESTATIONS}

Oral manifestations of CD include aphthous stomatitis, mucosal nodularity (cobblestoning), and pyostomatitis vegetans. Oral lesions occur in $10 \%$ of patients with CD and may represent the initial manifestation of disease.

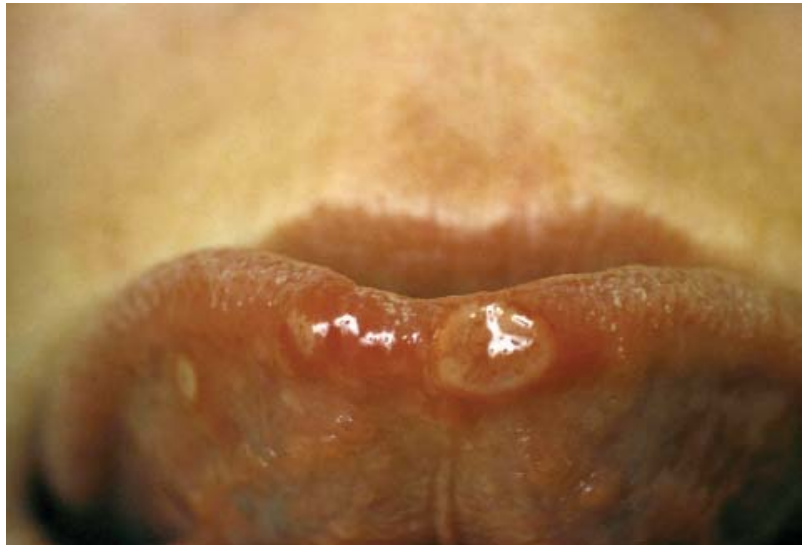

Figure 3 Aphthous stomatitis. Shallow, round ulcer with a central fibrinous membrane and erythematous halo.

\section{APHTHOUS STOMATITIS}

Aphthae are shallow round ulcers with a central fibrinous membrane and erythematous halo (see fig 3 ). The association of aphthae and IBD is well known. Aphthae associated with CD and UC cannot be differentiated clinically from common aphthae. ${ }^{6}$ Aphthae are also associated with celiac sprue, HIV/ AIDS, Behçet's disease, and Reiter's syndrome. Surprisingly, they occur less commonly in people who smoke or use snuff or chewing tobacco. ${ }^{51}$

The differential diagnosis includes oral herpes simplex, Behçet's disease, and coxsackievirus infection. Herpes simplex virus (HSV) lesions, although easily confused with aphthae in later stages, begin as vesicles that later ulcerate, while aphthae do not have a vesicular stage. When in doubt, Tzanck smear, antigen detection, serology, culture, or polymerase chain reaction to detect HSV can be used. Behçet's disease is an idiopathic vasculitis that causes oral and genital ulcers as well as ocular signs such as uveitis and iritis. Coxsackievirus lesions, like HSV, also begin as vesicles. $^{52}$

Treatment of the underlying condition may be curative. ${ }^{51}$ For symptomatic pain relief, $2 \%$ viscous lidocaine is frequently used. Treatment with topical corticosteroids such as triamcinolone $0.1 \%$ paste once to three times per day is effective at promoting healing. Dexamethasone elixir $0.5 \mathrm{mg}$ / $5 \mathrm{ml}$ swish and spit once to three times per day may also be beneficial. Finally, the non-steroidal anti-inflammatory paste amlexanox $5 \%$ can be applied to the ulcers to promote

\section{Key references}

- Berkowitz EZ, Lebwohl M. Cutaneous manifestations of inflammatory bowel disease. J Eur Acad Dermatol Venereol 2000;15:349-50.

- Lebwohl M, Lebwohl O. Cutaneous manifestations of inflammatory bowel disease. Inflamm Bowel Dis 1998;4:142-8.

- Powell F, O'Kane M. Management of pyoderma gangrenosum. Dermatol Clin 2002;20:347-55.

- Gonzales-Gay MA, Garcia-Porrua C, Pujol RM, et al. Erythema nodosum: a clinical approach. Clin Exp Rheumatol 2001;19:365-8.

- MacPhail L. Topical and systemic therapy for recurrent aphthous stomatitis. Seminars in Cutaneous Medicine and Surgery 1997;16:301-7. 
healing and reduce pain..$^{53}$ Systemic corticosteroids should only be used in refractory cases or in persistent or severe aphthae. ${ }^{54}$

\section{MUCOSAL NODULARITY (COBBLESTONING)}

Mucosal nodularity, known as cobblestoning, appears as mucosal coloured papules forming firm plaques on the buccal mucosa and palate. Lesions, although infrequent, are specific for CD. Cobblestoning may be painful and interfere with speaking and eating. ${ }^{55-57}$ Treatment of the underlying disease is necessary. In addition, systemic corticosteroids are effective, although topical corticosteroids can be used in less severe cases. ${ }^{58}$

\section{PYOSTOMATITIS VEGETANS}

Pyostomatitis vegetans is a rare oral ulcerative disorder seen in patients with UC and less frequently CD. Pustules, erosions, and vegetative plaques appear on the buccal and gingival mucosa and form a "snail track" appearance. Pyostomatitis vegetans is a specific marker for IBD. $^{59-61}$ Generally, IBD precedes the onset of pyostomatitis vegetans and mimics the activity of the bowel disease, ${ }^{60}$ although it has been reported with asymptomatic UC. ${ }^{62}$ Treating the underlying condition is essential and may cause resolution of the oral lesions. ${ }^{63}$ Topical corticosteroids have been useful in some cases, but the treatment of choice is systemic corticosteroids. Some have used systemic corticosteroids with dapsone or azathioprine, ${ }^{59}$ while one recent case report had success with cyclosporine A. ${ }^{61}$

\section{CONCLUSION}

Cutaneous manifestations of inflammatory bowel disease are common. Patients presenting with IBD should be examined for cutaneous manifestations. Treatment should be directed both at the cutaneous lesions and at the underlying systemic condition.

\section{MULIPLE CHOICE QUESTIONS (ANSWERS AT THE END OF THE REFERENCES)}

1. Which disease is associated with lesions that occur in response to minor trauma?
(A) Pyoderma gangrenosum
(A) Erythema nodosum
(B) Aphthous stomatitis
(C) Pyostomatitis vegetans

2. When treating pyoderma gangrenosum, which is generally considered the drug of choice?
(A) Debridement
(B) Potassium iodide
(C) Prednisone
(D) Alefacept

3. On which part of the body do the lesions of erythema nodosum classically occur?
(A) Trunk
(B) Hands
(C) Face
(D) Shins

4. Which is not a treatment for erythema nodosum?
(A) Leg elevation
(B) NSAIDs
(C) Potassium iodide
(D) Infliximab

5. What is one way that aphthous stomatitis may be distinguished from Behçet's disease?
(A) Aphthous stomatitis is associated with uveitis, iritis, and genital ulcers
(B) Behçet's disease is associated with uveitis, iritis, and genital ulcers
(C) Aphthous stomatitis is associated with ulcers on the shins
(D) Behçet's disease is associated with ulcers on the shins

\section{ACKNOWLEDGEMENTS}

We thank Melissa Praznovsky, Linda Roman, Flora Williams, and Tania Shulkin.

\section{Authors' affiliations}

L B Trost, J K McDonnell, Department of Dermatology, Cleveland Clinic Foundation, USA

Funding: none.

Competing interests: none.

\section{REFERENCES}

1 Loftus EV, Sandborn WJ. Epidemiology of inflammatory bowel disease. Gastroenterol Clin North Am 2002;31:1-20.

2 Podolsky DK. Inflammatory bowel disease. N Engl J Med 2002;347:417-29.

3 Danzi JT. Extraintestinal manifestations of idiopathic inflammatory bowel disease. Arch Intern Med 1988;148:297-302.

4 Berkowitz EZ, Lebwohl M. Cutaneous manifestations of inflammatory bowel disease. J Eur Acad Dermatol Venereol 2000;15:349-50.

5 Greenstein AJ, Janowiz HD, Sachar DB. The extraintestinal manifestations of Crohn's disease and ulcerative colitis: a study of 700 patients. Medicine 1976;55:401-12

6 Gregory B, Ho VC. Cutaneous manifestations of gastrointestinal disorders. Part II. J Am Acad Dermatol 1992;26:371-83.

7 Lebwohl $M$, Lebwohl $O$. Cutaneous manifestations of inflammatory bowel disease. Inflamm Bowel Dis 1998;4:142-8.

8 Powell FC, Su WRD, Perry HO. Pyoderma gangrenosum: classification and management. J Am Acad Dermatol 1996;34:395-409.

9 Bennett M, Jackson J, Jorizzo J, et al. Pyoderma gangrenosum: a comparison of typical and atypical forms with an emphasis on time to remission. Case review of 86 patients from 2 institutions. Medicine 2000;79:37-46.

10 Wollina U. Clinical management of pyoderma gangrenosum. Am J Clin Dermatol 2002;3:149-58.

11 Boh EE, Faleh al-Smadi RM. Cutaneous manifestations of gastrointestinal diseases. Dermatol Clin 2002;20:533-46.

12 Schwaegerle SM, Bergfeld WF, Senitzer D, et al. Pyoderma gangrenosum: a review. J Am Acad Dermatol 1988; 18:559-68.

13 Schorr-Lesnick B, Brandt U. Selected rheumatologic and dermatologic manifestations of inflammatory bowel disease. Am J Gastroenterol 1988;83:216-23.

14 Chow R, Ho V. Treatment of pyoderma gangrenosum. J Am Acad Dermatol 1996;34:1047-60.

15 Powell F, O'Kane M. Management of pyoderma gangrenosum. Dermatol Clin 2002;20:347-55.

16 Tamir A, Landau M, Brenner S. Topical treatment with 1\% sodium cromoglycate in pyoderma gangrenosum. Dermatology 1996;192:252-4.

17 Lyon C, Smith A, Griffiths C, et al. Peristomal dermatoses: a novel indication for topical steroids. J Am Acad Dermatol 2000;43:679-82.

18 Goldstein F, Krain R, Thornton J. Intralesional steroid therapy of pyoderma gangrenosum. J Clin Gastroenterol 1985;7:499-501.

19 Jennings J. Pyoderma gangrenosum: successful treatment with intralesional steroids. J Am Acad Dermatol 1983;9:575-80.

20 Powell FC, Collins S. Pyoderma gangrenosum. Clin Dermatol 2000;18:283-93.

21 Veloso FT, Carvalho J, Magro F. Immune-related systemic manifestations of inflammatory bowel disease: a prospective study of 792 patients. J Clin Gastroenterol 1996;23:29-34.

22 Matis WL, Ellis CN, Griffiths CEM. Treatment of pyoderma gangrenosum with cyclosporine. Arch Dermatol 1992;128:1060-4.

23 O'Donnell B, Powell FC. Cyclosporin treatment of pyoderma gangrenosum. J Am Acad Dermatol 1991;24:141-3.

24 Curley R, MacFarlane A, Vickers $C$. Pyoderma gangrenosum treated with cyclosporin A. Br J Dermatol 1985;113:601-4.

25 Penmetcha M, Navartnam AE. Pyoderma gangrenosum, response to cyclosporin A. Int J Dermatol 1988;27:253. 
26 Bijlmer-lest J, Rompelman-Schiere S, Van Ginkel C. Treatment of pyoderma gangrenosum with cyclosporin. Br J Dermatol 1991;135:283.

27 Schmitt EC, Pigatto PD, Boneschi V, et al. Pyoderma gangrenosum treated with low-dose cyclosporin. Br J Dermatol 1993;128:230-1.

28 Capella G, Frigerio E, Fracchiolla C, et al. The simultaneous treatment of inflammatory bowel diseases and associated pyoderma gangrenosum with oral cyclosporin A. Scand J Gastroenterol 1999;34:220-1.

29 Tutuncu Z, Morgan G, Kavanaugh A. Anti-TNF therapy for other inflammatory conditions. Clin Exp Rheumatol 2002;20:S146-51.

30 Grange F, Diilali-Bouzina F, Weiss A, et al. Corticosteroid-resistant pyoderma gangrenosum associated with Chrohn's disease: Rapid cure with infliximab. Dermatology 2002;205:278-80.

31 Ljung T, Staun $M$, Grove $O$, et al. Pyoderma gangrenosum associated with Crohn disease: Effect of TNF-alpha blockade with infliximab. Scand J Gastroenterol 2002;37:1 108-10.

32 Triantafillidis J, Cheracakis $P$, Sklavaina $M$, et al. Favorable response to infliximab treatment in a patient with active Crohn disease with pyoderma gangrenosum. Scand J Gastroenterol 2002;37:863-5.

33 Tan $M$, Gordon M, Lebwohl O, et al. Improvement of pyoderma gangrenosum and psoriasis associated with Crohn disease with anti-tumor necrosis factor alpha monoclonal antibody. Arch Dermatol 2001;137:930-3.

34 Hughes A, Jackson J, Callen J. Clinical features and treatment of peristomal pyoderma gangrenosum. JAMA 2000;284:1546-8.

35 Levitt $M$, Ritchie J, Lennard-Jones J, et al. Pyoderma gangrenosum in inflammatory bowel disease. Br J Surg 1991;78:676-8.

36 Mir-Madjlessi SH, Taylor JS, Farmer RG. Clinical course and evolution of erythema nodosum and pyoderma gangrenosum in chronic ulcerative colitis: a study of 42 patients. Am J Gastroenterol 1985;80:615-20.

37 White JW. Erythema nodosum. Dermatol Clin 1985;3:119-27.

38 Requena L, Requena C. Erythema nodosum. Dermatology Online Journal 2002;8:4.

39 Helm T. Skin lesions: when to suspect systemic causes. Cleve Clin J Med 1993;60:104

40 Gonzales-Gay MA, Garcia-Porrua C, Pujol RM, et al. Erythema nodosum: a clinical approach. Clin Exp Rheumatol 2001;19:365-8.

41 Apgar JT. Newer aspects of inflammatory bowel disease and its cutaneous manifestations: a selective review. Semin Dermatol 1991;10:138-47.

42 Fox MD, Schwartz RA. Erythema nodosum. Am Fam Physician 1992;46:818-22

43 Bullock WE. The clinical significance of erythema nodosum. Hosp Pract 1986;21:102E-102X

44 Brodell RT, Mehrabi D. Underlying causes of erythema nodosum-lesions may provide clue to systemic disease. Postgrad Med 2000;108:147-9.

45 Lehman CW. Control of erythema nodosum with naproxen. Cutis 1980;26:66-7

46 Ubogy Z, Persellin R. Suppression of erythema nodosum by indomethacin. Acta Derm Venereol 1982;62:265-6.
47 Elizaga FV. Erythema nodosum and indomethacin. Ann Intern Med 1982;96:383.

48 Horio T, Imamura S, Danno K, et al. Potassium iodide in the treatment of erythema nososum and nodular vasculitis. Arch Dermatol 1981;117:29-31.

49 Horio T, Danno K, Okamoto $\mathrm{H}$, et al. Potassium iodide in erythema nodosum and other erythematous dermatoses. J Am Acad Dermatol 1983;9:77-81.

50 Schulz EJ, Whiting DA. Treatment of erythema nodosum and nodular vasculitis with potassium iodide. Br J Dermatol 1976;94:75-8.

51 MacPhail L. Topical and systemic therapy for recurrent aphthous stomatitis. Seminars Cutaneous Medicine and Surgery 1997:16:301-7.

52 Shashy R, Ridley M. Aphthous ulcers: a difficult clinical entity. Am J Otolaryngol 2000;21:389-93.

53 Zunt S. Recurrent aphthous stomatitis. Dermatol Clin 2003;21:33-9.

54 McBride D. Management of aphthous ulcers. Am Fam Physician 2000:62:149-54.

55 Halme L, Meurman J, Laine P, et al. Oral findings in patients with active or inactive Crohn's disease. Oral Surg Oral Med Oral Pathol Oral Radiol Endod 1993;76:175-81.

56 Plauth $M$, Jenss $H$, Meyle J. Oral manifestations of Crohn's disease: an analysis of 79 cases. J Clin Gastroenterol 1991;13:29-37.

57 Lisciandrano D, Ranzi T, Carrassi A, et al. Prevalence of oral lesions in inflammatory bowel disease. Am J Gastroenterol 1996;91:7-10.

58 Williams A, Wray D, Ferguson A. The clinical entity of orofacial Crohn's disease. Q J Med 1991;79:451-8.

59 Soriano M, Martinez N, Grilli R, et al. Pyodermatitis-pyostomatitis vegetans: report of a case and review of the literature. Oral Surg Oral Med Oral Pathol Oral Radiol Endod 1999;87:322-6.

60 Calobrisi S, Mutasim D, McDonald J. Pyostomatitis vegetans associated with ulcerative colitis: temporary clearance with fluocinonide gel and complete remission after colectomy. Oral Surg Oral Med Oral Pathol Oral Radiol Endod 1995;79:452-4.

61 Brinkmeier T, Frosch P. Pyodermatitis-pyostomatitis vegetans: a clinical course of two decades with response to cyclosporine and low-dose prednisone. Acta Derm Venereol 2001;81:134-6.

62 Chaudhry S, Philpot N, Odell E, et al. Pyostomatitis vegetans associated with asymptomatic ulcerative colitis. Oral Surg Oral Med Oral Pathol Oral Radiol Endod 1999; 1999:327-30.

63 Storwick G, Prihoda M, Fulton R, et al. Pyodermatitis-pyostomatitis vegetans: a specific marker for inflammatory bowel disease. J Am Acad Dermatol 1994;31:336-41.

64 Das KM. Relationship of extraintestinal involvements in inflammatory bowel disease: new insights into autoimmune pathogenesis. Dig Dis Sci 1999:44:1-13.

ANSWERS

1. (A); 2. (C); 3. (D); 4. (D); 5. (B). 